\section{Online Biological Research Product Information and Sales}

\author{
David M. Sander, Ph.D., Sander \& Associates, \\ Consultants, Sacramento, CA (dmsander@ \\ virology.net)
}

The number of Web sites designed to serve scientists seems to have increased exponentially in recent years. $A$ variety of Web sites currently exist to help researchers with every step of the experimental process. These sites help researchers to find protocols for their experiments (e.g., NWFSC Molecular Biology Protocols, http:// research.nwfsc.noaa.gov/protocols.html) and to analyze their data (e.g., Metazoa, http://www.metazoa.com), and some even help researchers buy the reagents needed to perform the experiments. This last category of sites is the focus of this article.

Anyone who has ever played a role in keeping a laboratory supplied knows the challenges of the current laboratory product supply chain. To some extent, we have all spent time perusing shelves of hundreds of product catalogs, negotiating with salespeople and dealing with institutional bureaucracies and shipping regulations. Many scientists consider this to be a tremendous waste of valuable time, and these product purchase and information sites are here to help.

There are three subcategories of sites designed to aid scientists in the selection and purchase of products: (I) individual vendor sites that offer product information and purchasing, (ii) aggregation sites that help streamline the product purchasing process by grouping vendors and their products into one searchable database from which orders can be processed and (iii) comparison sites that offer information to help scientists decide which products to purchase.

\section{Vendor Information Sites}

It is now common for vendors of life science products to have a Web site that offers not only their catalog in digital format but also the option to purchase their products online. Many of these companies have spent a great deal of time and effort to ensure that their Web sites are both comprehensive and easy to use. The best vendor sites are easy to navigate, display pertinent product information prominently and do not require a user to tunnel deep within the site to get to the desired information.

One example of a well-designed vendor site is the New England Biolabs (NEB) site (http://www.neb.com). The NEB site is comprehensive and well organized, and it incorporates useful research features. One of its most impressive features is the REBASE database, which contains an all-inclusive collection of information on restriction enzymes (and methylases), including the recognition sequences, cleavage sites, methylation specificity, micro- organism of origin, commercial availability (including which vendors sell a particular restriction enzyme), references and published (as well as unpublished) laboratory observations. The NEB site also includes information on inteins (self-splicing proteins that can act as endonucleases and mediate DNA cleavage) and a section dedicated to coordinating genomic research on filarial nematode parasites. And of course, the site includes the NEB product catalog - the products of which can be purchased online through a secure Web interface.

To find other sites of this caliber, you will have to surf around and check out the sites of your favorite vendors. To aid your search, you may want to consult a meta-site that offers a collection of these vendor sites. For example, an extensive list of vendors and their Web sites can be found at the BioMall on BioTechniques's own site (http://www. biotechniques.com) or at the vendor site collection listed on my own site (http://www.virology.net/ garryfavwebcompan.html).

\section{Vendor Aggregators}

A major step towards simplifying the process of product procurement is the development of vendor aggregator sites. These sites have structured agreements with a number of different vendors that allow them to bring together enormous numbers of products from many different vendors at one site, thus creating a virtual marketplace. This area of product procurement is dominated by two large companies: Chemdex (now Ventro, http://www. chemdex.com) and SciQuest (http://www.sciquest. com), although other virtual distributors exist such as BioSupplies.com (http://www.biosupplies.com).

These sites have developed agreements with many vendors. As a result, these sites offer unprecedented access to products in one easy-to-peruse location on the Web. For example, SciQuest's Marketplace contains nearly a million products from more than 700 suppliers. To purchase products using these sites, the user must first register with the sites, providing the information that vendors would need to deliver a product (e.g., PO number, address and other contact information). Once this simple registration process is complete, users can search the site for a given product by either performing a text search or by scanning for the product by category or by supplier. Once the product is found, it can be selected and placed in an online shopping cart, allowing users to purchase a number of different products from different vendors all at one site. Obviously, this service can greatly simplify the ordering process and save a lot of valuable time.

These sites are also making great strides in integrating their services with leading procurement software packages used by their customers. This can be of great assistance to the researcher who must rely on an in-house institutional purchasing staff to actually make product purchasing arrangements. For example, at the Chemdex site, this integration allows their users to access the site from within their purchasing applications, to design price lists that reflect and use local pricing structures or institutional discounts, and to keep track of orders, invoices and 


\section{BioBit}

More sites with information about products or companies:

\section{Anderson's Webalog (http://www.atcg.com/aguide/ atcghome.htm)}

BioOnline (http://www.bio.com/os/start/home.html) Biospace (http://www.biospace.com)

HUM-MOLGEN (http://www.hum-molgen.de/ companies/index.html)

Yahoo! Biology Business Directory

(http://dir.yahoo.com/Business_and_Economy/

Business_to_Business/Scientific/Biology/)

Yahoo! Biotechnology and Drug Directory (http://biz. yahoo.com/p/_health-biotrx.html)

And some of the big suppliers:

Beckman Coulter (http://www.beckman.com/) Fisher Scientific (http://www.fisherscientific.com) Life Technologies (http://www.lifetech.com/) Millipore (http://www.millipore.com/) Perkin Elmer (http://www.perkinelmer.com/) Promega (http://www.promega.com) Sigma-Aldrich (http://www.sigma-aldrich.com)

Notes

Links to Web sites mentioned in this article along with other useful site links are available at the author's Web site (http://www.virology.net).

receive order status updates. These sites are also offering access to limited-time special offers and new product promotions. Among other interesting features offered by these sites are auctions that allow users to buy and sell used and refurbished laboratory equipment. (Yahoo has a similar service for business to business transactions: http://b2b.yahoo.com/26804216-category.html.)

There is a potential downside to these vendor aggregators, however, in that some vendors have exclusive deals with one virtual distributor. As a result, their products do not appear on the other distributors' sites. For example, Qiagen (Valencia, CA, USA), a company well known for nucleic acid purification kits, has an exclusive deal with SciQuest, and thus their products do not appear on the Chemdex site. This is the biggest drawback to these virtual marketplaces. Since one virtual distributor does not represent all companies, scientists searching for products at one of these sites may not have access to all product options.

\section{Product Information Sites}

Not all of the product-related Web sites are designed strictly for purchasing. There are sites that are designed to simply help scientists decide which product is right for their needs. An example is the recently launched Biocompare.com (http://www.biocompare.com). This site provides product information and consumer and professional reviews. It also alerts users to new technologies and promotions. Although Biocompare.com does not sell any products, it offers in-depth product information, giving users the opportunity to compare similar products from a number of different vendors. This, along with the reviews, allows researchers to make informed purchasing decisions.

Another site that falls into this category is Biowire (http:// www.biowire.com), which also provides consumer reviews of a variety of different products (although no product information is presented). Once registered with Biowire, users can read/write reviews and learn from the experiences of others.

An important aspect of these sites and others (e.g., Lablnternet, http://www.labinternet.com/; Laboratory Equipment, http://www.laboratoryequipment.com//) is that they allow users to determine what others in the scientific community think about a product. This information allows a scientist to make informed decisions when it comes to purchasing products, thus (hopefully) saving them time and money.

Other product information sites allow users to find out which companies produce products of interest. For example, LabVelocity (http://www.labvelocity.com) has created a system that allows users to search for and compare scientific research products by type and price. Besides product search tools, the site offers comparison tables, a collection of experimental protocols and commonly used reference tables, detailed maps of DNA vectors and software tools. Another useful site is BioMedWarehouse (http://www.biomedwarehouse.com/), which includes a searchable database of available products and prices.

The final member of this category-and a particularly useful site if you have not yet been convinced or been able to do all your product searching and purchasing online-BioSupplyNet (http://www.biosupplynet.com/) is a biomedical research supply information service that is operated with support from SciQuest. BioSupplyNet features a literature browser that allows users to request the latest catalogs and product literature (by old-fashioned mail in paper form) from many different suppliers. The site also has aids designed to help you find the companies that offer the supplies that you are seeking and has created an index (BioToolKit) of more than a thousand molecular biology research tools that can be found on the Web.

\section{Conclusion}

In the not-too-distant past, the only way to purchase life science products was to find a catalog, thumb through it to find the right product and then pick up the telephone to place the order. To do any sort of product comparison or to get user feedback, you had to walk up and down the hall and ask everyone you saw whether or not they had any experience with that product (and chances are you would have been out of luck). With the advent of these new Web sites, the process of finding the right product, choosing the vendor and making the purchase has been greatly simplified. 